\title{
Summary of Professor Shen's Experience in Treating Cough
}

\author{
Wenzhi Hao, Qian Chen, Feifei Xue* \\ School of Traditional Chinese Medicine, Jinan University, Guangzhou, China \\ Email: haowenzhi@126.com, ^fayexue@126.com
}

How to cite this paper: Hao, W.Z., Chen, Q. and Xue F.F. (2021) Summary of Professor Shen's Experience in Treating Cough. Journal of Biosciences and Medicines, 9 , 161-167.

https://doi.org/10.4236/jbm.2021.96015

Received: May 26, 2021

Accepted: June 20, 2021

Published: June 23, 2021

Copyright $\odot 2021$ by author(s) and Scientific Research Publishing Inc. This work is licensed under the Creative Commons Attribution International License (CC BY 4.0).

http://creativecommons.org/licenses/by/4.0/ (c) (i) Open Access

\begin{abstract}
Professor Shen pays attention to the simultaneous treatment of lung and stomach in the clinical treatment of cough. Professor Shen emphasized that the treatment of cough should be based on comprehensive judgments such as cough, phlegm, individual quality, general condition and pay attention to protecting the spleen and stomach while relieving cough. In the treatment, he is good at formulating prescriptions, using mulberry leaves and almonds, Uncaria and mint, Platycodonis Radix and Fritillaria thunbergii, Scutellariae Radix and Atractylodes macrocephala. Professor Shen pays attention to the impact of climate change on coughing. In the treatment of coughs occur in spring, he emphasizes on calming the liver and eliminating wind, nourishing yin and promoting body fluid while relieving the lung, relieving cough, resolving phlegm and relieving asthma. In the treatment of cough in autumn and winter, he focused on clearing, cooling and moisturizing, promoting body fluid and moisturizing. Nourish the viscera. Professor Shen inherited the style of ancient Lingnan medicine, and used the special herbal medicine of Lingnan area. He selected the prescriptions according to the locality. When treating coughs, he often used the combination of Seed of Indian Trumetflower, Roughhaired Holly Root, FoliumNerviliae, Herba Strigae, etc.
\end{abstract}

\section{Keywords}

Treatment of Lung and Stomach, Cough, Clinical Experience

\section{Introduction}

Professor Shen, Chief Physician, Famous TCM Doctor of Guangdong Province. Professor Shen has been engaged in clinical and teaching work for more than 50 years. He has been deeply influenced by the thoughts of physicians including $\mathrm{Li}$ Dongyuan, Huang Yuanyu, and Ye Tianshi. Combined with the characteristics 
of the Lingnan region, he has formed his own unique clinical academic thoughts creatively. In the clinical treatment of cough, he combines the characteristics of the Lingnan region with accurate syndrome differentiation, pays attention to the same treatment of the lung and stomach and is good at formulating the medicine, and makes good use of the Chinese herbal medicine of Lingnan. The author is currently treating patients in the clinic with Professor Shen, learning and summarizing the following experience of Professor Shen in treating cough.

\section{Simultaneous Treatment of Lung and Stomach Based on Syndrome Differentiation}

Professor Shen pays attention to the simultaneous treatment of lung and stomach in clinical treatment of cough, and treats according to symptoms and signs. Traditional Chinese medicine takes holistic concept as the outline, syndrome differentiation and treatment as the main point. It believes that the cause of cough is complicated. In the process of understanding and treatment, it is not limited to one organ and one organ, but from the overall perspective, which correlates the changes in the functions of the five organs and the six organs with the incidence of cough. In the five internal organs system, the dysfunction of gastric and lung is most closely related to cough, and the derived lung-stomach therapy is also an important point of clinical treatment of cough [1]. "Suwen of Yellow Emperor s Internal Classic" says: "(The cough of the five internal organs and six internal organs) are all related to the stomach and lungs, which clarifies the special position of the relationship between the lungs and stomach in the etiology and pathogenesis of cough, and establishes the program of syndrome differentiation and treatment. Later, based on the theory of the doctors of the Jin Dynasty, Yuan Dynasty, Ming Dynasty, and Qing Dynasty, the relationship between the lungs and stomach was further clarified. Liu Hejian believes that the essence of cough is the onset of internal injury of the spleen and stomach and externally induced wind pathogens. He believes that the spleen and stomach are rooted, and the lungs are the target. Later, Li Dongyuan further developed on this basis, emphasizing that lung qi injury is a cough, and spleen dampness is a cough, expounding the relationship between the spleen and stomach and lungs, and emphasizing that phlegm and Qi regulation is the key to cough treatment [2] [3]. On the basis of summarizing the basic experience of the predecessors, Huang Yuanyu created a Yuan Qi circulation theory to discuss the relationship between the lungs and stomachs. He believed that the dysfunction of the lungs and stomach interacting with each other can cause coughing. Professor Shen followed the principles of the predecessors, but did not stick to the ancient prescriptions. After summing up the thoughts of the previous generations of physicians, he formed his own unique clinical academic thinking, attaching importance to the simultaneous treatment of the lungs and stomach, and treating according to symptoms and signs. Professor Shen emphasized that the treatment of cough should be based on comprehensive judgments such as cough, phlegm, 
individual quality, and general condition. When relieving cough, pay attention to the spleen and stomach, especially the yin of stomach [4]. In clinical medications, Ophiopogon japonicus, Adenophora radicans, Poria cocos, licorice, tangerine peel, Pinellia, Scutellaria baicalensis and other drugs are often used, together with the addition and subtraction of drugs for relieving lungs and relieving cough.

\section{Good at Formulating Chinese Medicine Compound and Using Chinese Herbal Medicines}

TCM compound prescription is the characteristic of TCM syndrome differentiation and treatment, and it is also an important manifestation of TCM law following the evidence and formulating the law. In the clinical treatment of cough, Professor Shen is good at formulating prescriptions and using the right medicine, dialectically treating according to the different conditions of the patient [5]. Mulberry leaves and almonds, Uncaria and mint, Platycodonis Radix and Fritillaria thunbergii, Scutellariae Radix and Atractylodes macrocephala are the most commonly used medicine pairs for Professor Shen in the clinical treatment of cough.

Mulberry leaves are compatible with almonds, and the two are often used in conjunction with each other. Mulberry leaves are bitter and sweet in taste, slightly cold in nature, and belong to the lungs and liver meridians. "Medicinal Fu" says: "Mulberry leaves clears the liver and improves eyesight, good at eliminating wind-heat in the lungs and collaterals"; "Ben Jing Fengyuan"; "Mulberry leaves clears the lungs and stomach, removes wind and improves eyesight". Its nature is light, clear and divergent, and its effect is weak. The dosage should be large, generally $10-15 \mathrm{~g}$. Almonds taste bitter and warm, specialize in the Taiyin Lung Meridian. "Pearl sac tonic medicinal Fu" says: "almond moisturizes the lung dryness and relieves cough." Because of its small toxicity, the clinical dosage is small, generally not more than $10 \mathrm{~g}$.

Uncaria is compatible with mint, which is pungent and cold, returning to the lungs and liver meridian, it mainly radiates wind-heat, clears the throat, clears rashes and detoxification, soothes the liver and relieves depression. "Medicinal Fu" says: "Uncaria is Tasted pungent, cool in nature, non-toxic". Uncaria is sweet and cool in nature, which is returning to the liver and pericardium meridian, which can clear away heat and calm the liver, dispel wind and calm convulsions. Professor Shen chooses Uncaria to dispel wind and heat, relieve cough, relieve fever, relieve wind and liver, and treat children's cough. And repeated outstanding achievements in pregnancy cough. The usual dosages are $15 \mathrm{~g}$ for adults and $5 \mathrm{~g}$ for mint; $10 \mathrm{~g}$ for pregnant women and $5 \mathrm{~g}$ for mint; $5 \mathrm{~g}$ for children and $5 \mathrm{~g}$ for mint.

Platycodonis Radix is compatible with Fritillaria thunbergii, which tastes bitter and enters the lungs, and has the effect of resolving phlegm and relieving cough. Fritillaria thunbergii is cold in nature and enters the lung meridian, 
which mainly clears heat and relieves. "Compendium Supplements" says it: "Detoxification and phlegm, open lung qi, whoever has phlegm in the lungs is suitable for this." The "Shandong Handbook of Chinese Herbal Medicine" says: "Clear lungs, resolve phlegm, reduce acid, and detoxify. Treat colds and coughs, stomach pain, soreness, carbuncle, swelling and pain." Platycodonis Radix is bitter in nature, can rise and fall, can disperse and discharge, it can be the boat of various medicines, and it is also the guide of the lungs. Professor Shen believes that Platycodonis Radix and Fritillaria thunbergii can not only promote lung qi, but also reduce heat and reduce phlegm. The clinical effect is very good. The usual dosage is $10 \mathrm{~g}$ of Fritillaria thunbergii and $3-5 \mathrm{~g}$ of Platycodonis Radix.

The compatibility of Scutellariae Radix and Atractylodes macrocephala is Professor Shen's common medicine pair in the treatment of pregnancy cough. There are two main points in the treatment of pregnancy cough: cough relieving and tocolysis are the main points. In the Lingnan area, the climate is humid and hot, and the disease is often caused by dampness and heat. In particular, patients with cough during pregnancy often have the syndrome of spleen deficiency and dampness. Scutellariae Radix has a bitter and cold taste, enters the heart, lungs, gallbladder, and large intestine. The effect is to reduce the actual fire, remove dampness and heat, stop bleeding, and relieve the fetus. Atractylodes macrocephala is sweet and warm, enters the spleen and stomach meridian, it can rise and fall, and it can be used with Scutellariae Radix to relieve cough and tocolysis. The commonly used doses are $10 \mathrm{~g}$ of Scutellariae Radix and $15 \mathrm{~g}$ of Atractylodes macrocephala.

The compatibility of Gallus gallus domesticus and rice-grain Sprout is a common medicine used by Professor Shen in the treatment of children's cough. Gallus gallus domesticus is sweet and flat, enters the spleen and stomach meridian, can eliminate stagnation, invigorate the spleen and stomach, it is often used to treat malnutrition in children. "Materia Medica Renew" says it can: "resolve phlegm, regulate qi, and promote dampness." The rice-grain Sprout is sweet and flat in flavor and enters the spleen and stomach meridian. Its function is to eliminate food, dissolve accumulation, and invigorate the spleen and appetite. In the treatment of children's cough, Professor Shen often uses Gallus gallus domesticus and grain sprouts as a chinese drug pair. The commonly used dosage is $10 \mathrm{~g}$ of Gallus gallus domesticus and $30 \mathrm{~g}$ of grain sprouts.

\section{Use Lingnan Herbal Medicine}

In the treatment of cough, Professor Shen pays attention to the impact of environmental climate change and human physique on patients, and grasps the overall development of the disease. In terms of physical fitness, Professor Shen believes that people living in the Lingnan area are more like taking showers, and eating foods like raw frozen foods, fish, shrimps, seafood and other nourishing products, and the climate is humid and hot. Therefore, it is concluded that the patients' physique is Yin deficiency and damp-heat physique. In the treatment of 
cough, it is emphasized to differentiate the symptoms and treatments according to the different physique of the patient, and the tongue and pharynx are emphasized in the diagnosis, to clear heat and reduce dampness. In the treatment of four o'clock cough, Professor Shen pays attention to grasping the law of the season, and conducts syndrome differentiation treatment according to the characteristics of different seasons. In the treatment of coughs that occur in the spring, Professor Shen pays attention to calming the liver and eliminating wind, nourishing yin and promoting body fluid while promoting the lungs, relieving cough, phlegm and asthma. When coughing in spring, it is often used to add and subtract Sangju Decoction, compatible with Scutellaria baicalensis, Uncaria, Artemisia annua, etc. to clear away heat and liver, and compatible with Reed Root, Trichosanthes to nourishing body fluids. The autumn and winter seasons are characterized by coldness and dryness. Therefore, in the treatment of coughs occur in autumn and winter, Professor Shen focused on clearing, cooling, moisturizing, promoting body fluid and nourishing internal organs while relieving cough. The clinical prescriptions are often supplemented with Jinshui Liujun Decoction and Xiangsha Liujunzi Decoction to nourish the spleen and kidneys, in which Beisha is often used for cleansing the lungs and moisturize dryness.

Professor Shen also emphasized that when treating cough by differentiation of symptoms and signs, it is necessary to adapt measures to local conditions, combine local characteristics, and use local herbal medicines. The Lingnan area is the southern part of Yuechengling, Dupangling, Mengzhuling, Qitianling, and Dayuling five ridges, and the climate is mainly hot and humid. Professor Shen followed the insights of ancient physicians and had a deep understanding of the hot and humid characteristics of the Lingnan area. When treating cough, he pays attention to clearing away heat and relieving cough, resolving phlegm and dampness, protecting stomach yin, promoting body fluid and moisturizing dryness. Professor Shen inherited the style of ancient Lingnan medicine, used the special herbal medicine of Lingnan area, and selected the prescriptions according to the locality. In the treatment of cough, he often chose the Seed of Indian Trumetflower, Roughhaired Holly Root, FoliumNerviliae, Herba Strigae, etc. [6]. Seed of Indian Trumetflower has a sweet and cool nature. It is good at clearing the lungs and relieving the pharynx. It is mainly used to treat aphonia and dry dumbness. It has repeated miraculous effects in the clinical treatment of cough and dumbness of the throat. The general medication is $10 \mathrm{~g}$; Roughhaired Holly Root tastes bitter, sweet and cold, and it is mainly used to clear heat and produce fluid. The "Lingnan Collection of Medicines" says: "clearing heat and toxins. Decoction of herbal tea is used for many purposes". It can be used in conjunction with the treatment of cough and swollen tonsils. The general dosage is $15 \mathrm{~g}$; Qingtian Kui (FoliumNerviliae) comes from "Lingnan Collection of Medicines". It is sweet in taste and has the effect of clearing away heat, nourishing lungs and dispelling blood stasis, regulating phlegm and hemoptysis. Professor Shen is generally used in the treatment of fiery cough with blood; Herba Strigae is sweet, 
light and cool in nature and flavor, which is used for clearing away heat and eliminating accumulation. It is generally used in the treatment of cough due to food accumulation in children, with amazing results.

\section{Clinical report}

\section{Report 1:}

Ms. Liang, female, 52 years old. First visit on July 7, 2020. The patient complained of repeated coughing for 20 years. The cough was continuous during the attack period, sometimes with or without sputum, accompanied by dry throat and red throat, discomfort on both sides, and stool 2 - 3 times a day. The patient currently has chronic superficial gastritis with multiple ulcers, and the appetite is acceptable. Check the tongue and see the tongue is red and white, and the pulse string is fine. The dialectical is the deficiency of lung and kidney yin, the prescription is Jinshui Liujun decoction.

Rehmannia glutinosa 20 g, Poria 15 g, French Pinellia 10 g, Tangerine peel 10 g, Roasted licorice $5 \mathrm{~g}$.

Zhe Fritillaria $10 \mathrm{~g}$, Platycodon grandiflorum 5g, Ophiopogon japonicus $10 \mathrm{~g}$, White front $10 \mathrm{~g}$, Uncaria $15 \mathrm{~g}$.

7 doses, decocted in water.

Second diagnosis: The patient complained that he had blood in his mouth early in the morning after taking the medicine, and his tongue and pulse were the same as before. Add $15 \mathrm{~g}$ of Eclipta prostrata and $6 \mathrm{~g}$ of Rhodiola to the first prescription.

Note: The TCM diagnosis of this disease is cough, and the syndrome belongs to deficiency of lung and kidney yin. At the first diagnosis, the patient had self-reported cough for more than 20 years. During the onset, the cough was continuous, sometimes there was sputum but no sputum, accompanied by dry throat and red throat. It was a sign of lung yin deficiency, dystrophy of lung collaterals, and endogenous deficiency and heat. The lungs are delicate viscera. It is need to be clear and moisturize but not to be dry. Professor Shen uses Jinshui Liujun Decoction as the main recipe to nourish the lungs and kidneys.

\section{Report 2:}

Ms. Zhu, female, 34 years old. Cough and sputum for 2 days, thick yellow sputum. The pregnancy has been dicovered for five months. The tongue is red and the coating is thin, with tooth marks and slippery pulse strings. The dialectic is wind-heat cough, and the prescription is treated with Sangju.

Mulberry leaves 10 g, Platycodon grandiflorum 5 g, Zhebei 10 g, Scutellaria baicalensis Georgi $10 \mathrm{~g}$, Baizhu $15 \mathrm{~g}$.

Windproof $10 \mathrm{~g}$, Mint $5 \mathrm{~g}$, Uncaria $10 \mathrm{~g}$, Licorice $5 \mathrm{~g}$.

5 doses, decocted in water.

Note: The TCM diagnosis of this disease is cough, and the syndrome is wind-heat cough. The patient coughed and sputum for two days, the sputum was thick and yellow, which was a symptom of wind-heat cough. Professor Shen 
used Sangju Decoction as the prescription, and used Zhebei and Platycodon grandiflorum flavor to promote lung qi and lower heat to reduce phlegm. Considering that the patient's pregnancy is more than five months old, the tongue has tooth marks. Professor Shen mainly focuses on relieving cough and resolving phlegm. Therefore, the combination of Scutellaria baicalensis and Atractylodes has the effect of relieving cough and relieving fetus. And the combination of Uncaria mint, dispelling wind and heat, relieving the throat and relieving cough.

\section{Acknowledgements}

This research was supported by the National Natural Science Foundation of China (No. 82074331) and the sixth batch of national special funds for the academic experience of old Chinese medicine experts (34018101).

\section{Conflicts of Interest}

The authors declare no conflicts of interest regarding the publication of this paper.

\section{References}

[1] Zhu, P.Y., Su, G., Jiang, Y. and Cao, Y. (2014) Discussing the Related Mechanisms of the Lung and Stomach of TCM from the Perspective of Nature and Man. Chinese Journal of Basic Medicine in Traditional Chinese Medicine, 20, 567-569.

[2] Zou, J.H., Xiao, Z.S. and Wang, S.C. (2019) Discussion on the Application of the Theory of Lung and Spleen-Stomach Deficiency in Piwei Lun in Pediatric Chronic cough. China Journal of Traditional Chinese Medicine and Pharmacy, 34, 4096-4098.

[3] Song, Y.Z. (2017) Research on Li Dongyuan's Academic Thoughts in Treating Asthma. Guangzhou University of Traditional Chinese Medicine, Guangzhou.

[4] Wang, Y.J. and Ma, J. (2016) Analysis of Ye Tianshi's Differentiation and Treatment of Chronic Cough from Spleen and Stomach. Journal of Beijing University of Traditional Chinese Medicine, 39, 545-546.

[5] Zhang, J., Sun, S.Y. and Ge, M.G. (2011) Examples of Shen Yingsen's Commonly Used Drugs. Liaoning Journal of Traditional Chinese Medicine, 38, 962-963.

[6] Zhu, S.P., Feng, W.F. and Shen, Y.S. (2017) Professor Shen Yingsen's Academic Thoughts on Treating Cough in Children. Journal of Yunnan University of Traditional Chinese Medicine, 40, 95-97. 